Pontifícia Universidade $_{\text {datólica }}$

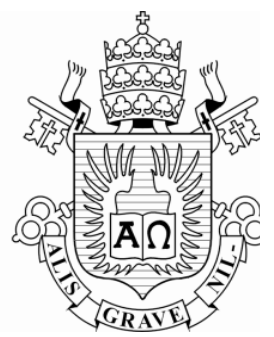

Yovanna Gisela Palomares Yallico

\title{
Reatividade Comparativa de Coque, Carvão mineral, Carvão Vegetal e Coque Verde de Petróleo
}

\section{Dissertação de Mestrado}

Dissertação apresentada como requisito parcial para obtenção do título de Mestre pelo Programa de PósGraduação em Engenharia de Materiais e Processos Químicos e Metalúrgicos da PUC - Rio como requisito parcial para a obtenção do título de Mestre em Engenharia de Materiais e de Processos Químicos e Metalúrgicos.

Orientador: Prof $^{\circ}$. Jose Carlos D'Abreu 


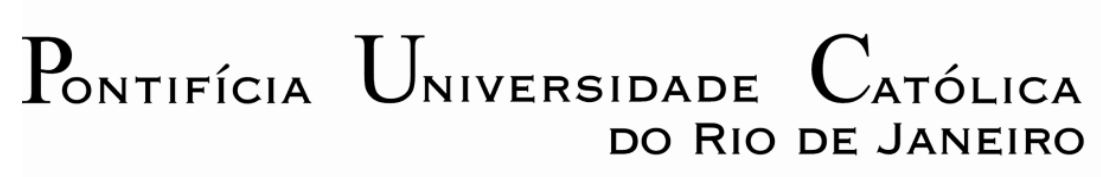

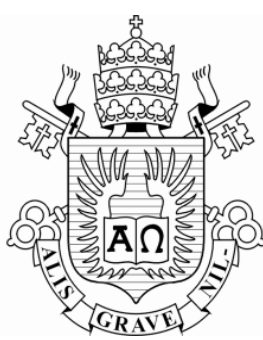

Yovanna Gisela Palomares Yallico

\section{Reatividade Comparativa de Coque, Carvão Mineral, Carvão Vegetal e Coque Verde de Petróleo}

Dissertação apresentada como requisito parcial para obtenção do título de Mestre pelo Programa de Pós-Graduação em Engenharia de Materiais e de Processos Químicos e Metalúrgicos da PUC - Rio. Aprovada pela Comissão Examinadora abaixo assinada.

Prof ${ }^{\circ}$. Jose Carlo D'Abreu

Orientador e presidente PUC - Rio

Prof $^{\circ}$. Francisco Jose Moura Departamento de Engenharia de Materiais- PUC - Rio

Prof $^{\circ}$. Helio Marques Kolher Samarco Mineração, SAMARCO - Brasil

Prof $^{\circ}$. José Eugênio Leal Coordenador Setorial do Centro Técnico Científico - PUC - Rio

Rio de Janeiro, 9 de Setembro de 2011 
Todos os direitos reservados. É proibida a reprodução total ou parcial do trabalho sem autorização da universidade, do autor e do orientador.

Yovanna Gisela Palomares Yallico

Bachiller em Engenharia Metalúrgica pela Universidad Nacional de Ingeniería em Perú.

\section{Ficha Catalográfica}

\section{Palomares Yallico, Yovanna Gisela}

Reatividade comparativa de coque, carvão mineral, carvão vegetal, e coque verde de petróleo / Yovanna Gisela Palomares Yallico; orientador: José Carlos D'Abreu. - 2011.

108 f.: il. (color.); $30 \mathrm{~cm}$

Dissertação (mestrado)-Pontifícia Universidade Católica do Rio de Janeiro, Departamento de Engenharia de Materiais, 2011.

Inclui bibliografia

1. Engenharia de materiais - Teses. 2. Reatividade. 3. Carvão. 4. Coque. 5. Carvão vegetal. I. D’Abreu, José Carlos. II. Pontifícia Universidade Católica do Rio de Janeiro. Departamento de Engenharia de Materiais. III. Título. 
Dedico este trabalho a meus avós Felícia e Fortunato 


\section{Agradecimentos}

Por toda a vida agradeço a Deus por eu ser feliz, saudável e ter uma família e amigos verdadeiros.

Aos professores Jose Carlos D’Abreu, Helio Marques Kohler, pela orientação e apoio para a realização deste trabalho, verdadeiros conselheiros para minha vida profissional e pessoal

A minha mãe, Lidia, e meu pai, Jesus, sempre me motivando e com seus apoios para que buscasse atingir meu sonhos, pelo apoio constante, carinho e preocupação.

A meu amigo Raimundo Nonato, pela ajuda nas práticas laboratoriais e por sua paciência na aprendizagem do português.

Ao grupo de trabalho de pesquisa em Siderurgia, pelo suporte nas horas que precisei e por oferecer também seus conhecimentos.

Ao CNPq e À PUC - Rio, pelo apoio concedido para a realização desta Dissertação.

A todos os professores, funcionários e colegas do Departamento de Engenharia de Materiais e Processos Químicos e Metalúrgicos. 


\section{Resumo}

Palomares Yallico, Yovanna Gisela; D’Abreu, Jose Carlos; Reatividade comparativa de coque, carvão mineral, carvão vegetal e coque verde de petróleo. Rio de Janeiro, 2011. 108 p. Dissertação de Mestrado - Departamento de Engenharia de Materiais. Pontifícia Universidade Católica do Rio de Janeiro.

A Indústria siderúrgica, visando contribuir com a minimização das emissões de $\mathrm{CO}_{2}$, têm promovido o estudo de vários materiais carbonosos, um deles sendo a alternativa relacionada à utilização do carvão vegetal como fonte renovável. O objetivo principal desse trabalho foi medir, em escala de laboratório, a reatividade ao $\mathrm{CO}_{2}$ dos materiais carbonosos, em particular o uso do carvão vegetal em comparação com o mineral, o coque metalúrgico e o coque verde de petróleo, utilizando para tal o método de perda de peso (ASTM D5341-99). O processo se realizou usando briquetes de cada material, previamente cominuido a um tamanho de partícula menor que $125 \mu \mathrm{m}$, levados a um forno tubular a uma temperatura de trabalho de $1100{ }^{\circ} \mathrm{C}$, com injeção de $\mathrm{N}_{2}$ para manter uma atmosfera inerte apenas no inicio e no final dos ensaios, sob um fluxo de $0,6 \mathrm{Nl} / \mathrm{min}$, visando as etapas de aquecimento e resfriamento. Utilizando como agente oxidante 0 $\mathrm{CO}_{2}$ durante $2 \mathrm{~h}$, com um fluxo de $0,9 \mathrm{NI} / \mathrm{min}$, todos os tipos de briquetes foram tratados na temperatura do ensaio $\left(1100^{\circ} \mathrm{C}\right)$. Os resultados obtidos mostraram que o carvão vegetal foi o que apresentou maior reatividade e o coque verde de petróleo a menor, entre todos os materiais ensaiados, tanto para os briquetes não desvolatilizados como desvolatilizados. Quanto ao carvão mineral e o coque, eles situaram suas reatividades intermediariamente, ficando o carvão mineral com maiores valores de reatividade do que o coque, tanto no estado desvolatilizado como no não desvolatilizado.

\section{Palavras-chave}

Reatividade; Carvão Mineral; Coque; Carvão Vegetal; Coque Verde de Petróleo. 


\section{Abstract}

Palomares Yallico, Yovanna Gisela; D’Abreu, José Carlos (Advisor); Comparative Reactivity of Coke, Coal, Charcoal and Green Petroleum Coke. Rio de Janeiro, 2011.108p. MSc. Dissertation - Departamento de Engenharia de Materiais, Pontifícia Universidade Católica do Rio de Janeiro.

The steel industry contributes to the minimization of emissions of $\mathrm{CO}_{2}$ promoting the study of carbonaceous materials, one of them being the charcoal, a renewable source. The main objective of this study was to measure, in a laboratory scale, selected carbonaceous materials reactivity for $\mathrm{CO}_{2}$. To perform it, charcoal was elected to be compared with coal, metallurgical coke and green petroleum coke (pet coke). The quantitative results were obtained by the method of weight loss (ASTM D5341-99). The procedure was carried out using briquettes of each material, previously grinded to a particle size smaller than $125 \mu \mathrm{m}$, and fed to a tubular furnace at a temperature of $1100^{\circ} \mathrm{C}$, using a $\mathrm{N}_{2}$ injection, with a stream of $0.6 \mathrm{NI} / \mathrm{min}$, to insure an inert atmosphere only during heating and cooling steps of the experiments. To perform the reactivity tests, $\mathrm{CO}_{2}$ was used as the species to react with the $\mathrm{C}$ element present at the samples composition. The tests took 2 hours, with a $\mathrm{CO}_{2}$ flow rate of $0.9 \mathrm{NI} / \mathrm{min}$, in a temperature of $1100^{\circ} \mathrm{C}$ According to the results obtained, it was found that the charcoal has the higher reactivity and the green petroleum coke the less reactive. For the metallurgical coke and coal, their reactivities were intermediary between charcoal and green pet coke, being coal more reactive than coke for both, volatilized and non-volatilized samples.

\section{Keywords}

Reactivity; Coal; Coke; Charcoal; Green Petroleum coke. 


\section{Sumário}

1. Introdução 15

1.1. Alto-Forno 16

1.1.1. Características principais de cada zona interna do Alto Forno 17

1.1.1.1. Zona Granular 17

1.1.1.2. Zona Coesiva ou Zona de Amolecimento e Fusão (1000 a $\left.1450^{\circ} \mathrm{C}\right) \quad 18$

1.1.1.3. Zona de Gotejamento $\left(1400^{\circ} \mathrm{C}\right.$ a $\left.1800^{\circ} \mathrm{C}\right)$

1.1.1.4. Zona de Combustão $\left(2000^{\circ} \mathrm{C}\right)$

1.1.1.5. Zona do Cadinho 22

1.1.2. Reações internas no Alto Forno 22

1.1.3. Importância da Reação de Boudouard no alto forno 23

1.1.3.1. Efeito da Pressão sobre a Reação de Boudouard 25

1.1.3.2. Cinética da reação de Boudouard 26

1.1.3.3. Diagrama de Chaudron na redução de óxidos de Ferro e a Reação de

Boudouard. 27

2. Objetivos 31

2.1. Objetivo geral 31

2.2. Objetivos específicos 31

3. Revisão Bibliográfica 32

3.1. Reatividade 32

3.1.1. Definição de Reatividade 32

3.1.2. Fatores que influenciam a Reacao de Boudouard 33

3.1.3. Reatividade de Carvão em Redução Direta 38

3.1.4. Determinação da Função de perda de Massa por reação 40

3.1.5. Cálculo da Reatividade Específica 42

3.2. Carvão Mineral 45

3.2.1. Definição 45

3.2.2. Origem e Formação 46

3.2.2.1. Carbonificação 46

3.2.3. Eras e Períodos na Formação do Carvão 48

3.2.4. Classificação do carvão 49 
3.2.5. Caracterização de carvão para a Fabricação de Coque de Alto Forno. 51

3.2.5.1. Análises Imediatas: 51

3.3. Carvão Vegetal 54

3.3.1. Introdução na Siderurgia 54

3.3.2. Matéria Prima para o Carvão Vegetal 56

3.3.2.1. Madeira Nativa 56

3.3.2.2. Madeira de Reflorestamento 56

3.3.3. Fabricação de Carvão Vegetal 57

$\begin{array}{lll}\text { 3.3.3.1. Pirólise } & 57\end{array}$

3.3.3.2. Carbonização $\quad 57$

3.3.4. Produtos obtidos na pirólise lenta ou carbonização controlada 59

3.3.5. Caracterização de Carvão Vegetal. 62

3.3.5.1. Porosidade 62

3.3.5.2. Composição Química 63

3.3.5.3. Densidade Aparente 63

3.3.5.4. Umidade 63

3.3.5.5. Resistência mecânica 64

3.3.5.6. Granulometria 64

3.3.5.7. Carbono fixo 65

3.3.5.8. Materiais Voláteis 65

3.3.5.9. Cinzas 65

$\begin{array}{lll}3.3 .5 .10 . & \text { Reatividade } & 65\end{array}$

3.4. Coque Metalúrgico 68

3.4.1. Definição 68

3.4.2. O coque no Alto Forno 68

3.4.3. Controle da qualidade do coque metalúrgico 69

$\begin{array}{lll}\text { 3.4.3.1. Propriedades físicas } & 70\end{array}$

3.4.3.2. Propriedades químicas 72

3.4.4. Coqueificação 73

3.4.5. Novas tendências no controle da qualidade do coque, reatividade 73

$\begin{array}{ll}\text { 3.4.5.1. Reatividade } & 73\end{array}$

3.5. Coque Verde de Petróleo 74

$\begin{array}{ll}\text { 3.5.1. Definição } & 74\end{array}$

3.5.2. Processo de obtenção de Coque verde de Petróleo 76

$\begin{array}{ll}\text { 3.5.3. Produção de coque verde de petróleo. } & 77\end{array}$

$\begin{array}{ll}\text { 3.5.4. Aplicações } & 79\end{array}$

3.5.4.1. Como Redutor Metalúrgico 79 
3.5.4.2. Como Combustível Sólido 80

3.5.4.3. Outras aplicações na Indústria Siderúrgica: 80

4. Desenvolvimento Experimental 82

4.1. Materiais e Equipamentos 82

4.1.1. Caracterização dos Materiais Utilizados 82

4.1.1.1. Coque Verde de Petróleo. 82

4.1.1.2. Coque Verde de Petróleo. 82

4.1.1.3. Carvão Vegetal 83

4.1.1.4. Carvão Mineral 83

4.1.1.5. Outros Materiais $\quad 84$

4.1.2. Equipamentos e Aparelhos Usados 84

4.1.3. Preparação das Amostras 84

4.1.3.1. Material não desvolatilizado $\quad 87$

$\begin{array}{ll}\text { 4.1.3.2. Material Desvolatilizado } & 91\end{array}$

5. Resultados e Discussões 92

5.1. Resultados na realização dos briquetes 92

5.2. Resultados para as amostras não desvolatizadas 93

5.3. Resultados com as amostras desvolatizadas 95

5.4. Resultado de Reatividade das amostras não desvolatilizadas 96

5.5. Resultado de Reatividade das amostras desvolatilizadas 97

5.6. Comparação de Reatividade de amostras não desvolatilizadas e $\begin{array}{ll}\text { desvolatilizadas. } & 97\end{array}$

6. Conclusões 99

7. Referências bibliográficas 100

$\begin{array}{ll}\text { 8. Apêndice } & 105\end{array}$ 


\section{Lista de figuras}

Figura 1 - Zonas de Alto-Forno, b) atmosfera gasosa na redução do minério de ferro de um alto-forno. (Fonte: MOURÃO, M. 2007)

Figura 2 - Escoamento (a) situação ideal para passagem gasosa; (b) Situação não ideal para passagem gasosa devido ao acumulo de finos (Fonte: Arcelor Mittal Tubarão).

Figura 3 - Formação da estrutura da camada coesiva do alto forno (Fonte: PIMENTEL, F. et al. 2007).

Figura 4 - Esquema da distribuição de carga da Zona de amolecimento e fusão (zona coesiva) em um alto forno (PIMENTEL, F. et al. 2007).

Figura 5 - Esquema de uma seção vertical a uma ventaneira de alto forno.

Figura 6- Disposição das cargas no interior do alto-forno (Fonte: Adaptado de ARAÚJO, L. 2008)

Figura 7 - Sistema C-O, Curva de Boudouard (Fonte: PIMENTEL, F.et.al (2007).

Figura 8 - Diagrama de Equilíbrio Fe-C-O indicando a composição dos gases. (Fonte: CAMPOS,V. 1984)

Figura 9 - Efeito da variação na pressão sobre o equilíbrio da reação de Boudouard 26

Figura 10- Diagrama de Chaudron (Oxi-redução)

Figura 11 - Leito de Coque (SHIFINO, 1977)

Figura 12 - Representação esquemática das macromoléculas do carvão ((SHUMANN, W.1985)

Figura 13 - Processo da carbonificação (Fonte: Uma publicação por World coal Institute, 2007)

Figura 14 - Eras e períodos geológicos: "possibilidade de formação de carvão ao longo de diferentes eras e períodos" (ULHOA, 1999)

Figura 15 - Tipos de carvões e principais usos (Fonte: Agencia Nacional de Energia Elétrica, ANEEL).

Figura 16 - Tipos e usos do carvão mineral (Fonte: Empresa de Pesquisa Energética, EPE) 
Figura 17 - Exemplo típico de emissões específicas de $\mathrm{CO}_{2}, \mathrm{O}_{2}$ e $\mathrm{SO}_{2}$, para a produção integrada de ferro gusa com carbono não renovável fóssil ,kg/ $\mathrm{t}$ ferro gusa (Fonte: Oliveira,L.2010)

Figura 18 - Balanço de $\mathrm{CO}_{2}$ e $\mathrm{CO}_{2}$ a produção de ferro gusa via uso de biomassa renovável ,kg/t de ferro gusa (Fonte: Oliveira,L. 2010).

Figura 19 - Fluxograma de emissão de gases de produção de aço liquido pela rota de coque e carvão vegetal. (Fonte: FERREIRA, O. 2000)

Figura 20 - Analise termogravimétrica da Madeira e seus componentes (BRAGA,R.1992)

Figura 21 - Esquema do fluxo de produção e aplicação do coque verde de petróleo

Figura 22 - Fluxograma simplificado de unidade de destilação

Figura 23 - Principais utilizações e correspondentes qualidades de coque verde petróleo.

Figura 24 - Rotap, (Fonte DEMa- PUC-Rio)

Figura 25 - Moinho de Porcelana, (Fonte DEMa - PUC-Rio)

Figura 26 - Gral de Porcelana, (Fonte DEMa- PUC-Rio)

Figura 27 - Matriz de (Fonte DEMa- PUC-Rio)

Figura 28 - Realização dos briquetes: (a) Entrada do molde menor; (b) Colocar a amostra; (c) Colocar o molde maior; (d) Pressionar o molde; (e) Pressão a 1000kgf; (f) briquetes cilíndricas feitos.

Figura 29 - Forno Tubular (Esquerda); Amostras de coque verde de petróleo colocada ao inicio no forno tubular (Direita)

Figura 30 - Figura Coque verde de petróleo depois da saída do forno

Figura 31 - Forno Tubular (Esquerda); Amostras de carvão mineral colocada ao inicio no forno tubular (Direita).

Figura 32 - Carvão mineral depois da saída do forno

Figura 33 - Forno Tubular (Esquerda); Amostras de carvão vegetal colocada ao inicio no forno tubular (Direita).

Figura 34 - Carvão vegetal depois da saída do forno

Figura 35 - Forno Tubular (Esquerda); Amostras de coque metalúrgico colocada ao inicio no forno tubular (Direita).

Figura 36 - Coque Metalúrgico depois da saída do forno.

Figura 37 - Esquema comparativo das amostras ensaiadas de Reatividade 


\section{Lista de tabelas}

Tabela 1-Variação das propriedades físicas e metalúrgicas do carvão de acordo com o grau de carbonização.(COSTA,M.2002)

Tabela 2-Composição Química dos combustíveis Fósseis Sólidos. (ROBERTO,F. 2001)

Tabela 3-Principais Componentes da Cinza \%, Fonte: BRIGDA, 1995)

Tabela 4-Produtos de carbonização (Fonte: PIMENTA, A. 2002)

Tabela 5-Análise Química Imediata do Carvão Vegetal e Rendimento em Carbono Fixo (Fonte CETEC)

62

Tabela 6-Influencia da temperatura de carbonificação nas propriedades químicas e física do carvão vegeta, (Fonte: Adaptado por Oliveira,et al.1982)

Tabela 7-Composição elementar do carvão vegetal e rendimento em relação à madeira seca em função da temperatura de carbonização (Fonte: Adaptado por Bergstrom, 1978)

Tabela 8-Influencia da temperatura de carbonização sobre o limite de resistência à compressão (Fonte: Pelatorio de projeto CETEC,1978)

Tabela 9-Influência da temperatura de carbonização sobre a reatividade do carvão (Fonte: CETEC)

Tabela 10-Carvões Coqueificaveis

Tabela 11-Requisitos físicos e químicos de carvão metalúrgico (ABNT) 70

Tabela 12-Analise imediata do Coque Verde de Petróleo

Tabela 13-Analise imediata do Coque Verde de Petróleo

Tabela 14- Características gerais do CVP da Petrobras.

Tabela 15- Aplicações de Coque verde de Petróleo 81

Tabela 16-Analise Imediata de coque verde de petróleo 82

Tabela 17-Analise Imediata de Coque Metalúrgico 83

Tabela 18-Analise Imediata de carvão vegetal 83

Tabela 19- Analise Imediata do Carvão Mineral 83

Tabela 20- Parâmetros de ensaio com Coque Verde de Petróleo 88

Tabela 21- Parâmetros de ensaio com Carvão Mineral 89

Tabela 22-Parâmetros de ensaio com Carvão Vegetal 90 
Tabela 23- Parâmetros de ensaio com Coque Metalúrgico 91

Tabela 24- Porcentagem de Finos das amostras 92

Tabela 25- Comportamento dos materiais na pressão de 1000kgf 92

Tabela 26-Comportamento dos materiais com um incremento de ligante e água de $2 \%$ com uma pressão de 1000kgf 92

Tabela 27- Comportamento dos materiais com um incremento de ligante e água de $2 \%$,com uma pressão de 1000kgf 93

Tabela 28- Quantidade empregada no teste. 93

Tabela 29- Condições de trabalho e resultados feitos para a amostra não desvolatilizada de Coque Metalúrgico.

Tabela 30- Condições de trabalho e resultados feitos para a amostra não desvolatilizada de Carvão Mineral

Tabela 31- Condições de trabalho e resultados feitos para a amostra não desvolatilizadas de Carvão Vegetal.

Tabela 32- Material volátil dos materiais ensaiados

Tabela 33- Condições de trabalho e resultados feitos para amostra desvolatilizada de coque verde de petróleo

Tabela 34- Condições de trabalho e resultados feitos para amostra desvolatilizada de coque metalúrgico

Tabela 35- Condições de trabalho e resultados feitos para amostra desvolatilizada de carvão mineral

Tabela 36- Condições de trabalho e resultados feitos para amostra desvolatilizada de carvão vegetal

Tabela 37- Reatividade das amostras não desvolatilizadas

Tabela 38- Reatividade das amostras desvolatilizadas

Tabela 39- Comparação de reatividade para cada material 